\title{
CRAWFORD NUMBERS OF COMPANION MATRICES
}

\author{
Hwa-Long GaU, KuO-Zhong Wang And PeI Yuan Wu
}

Abstract. The (generalized) Crawford number $C(A)$ of an $n$-by- $n$ complex matrix $A$ is, by definition, the distance from the origin to the boundary of the numerical range $W(A)$ of $A$. If $A$ is a companion matrix

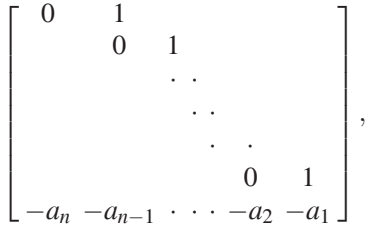

then it is easily seen that $C(A) \geqslant \cos (\pi / n)$. The main purpose of this paper is to determine when the equality $C(A)=\cos (\pi / n)$ holds. A sufficient condition for this is that the boundary of $W(A)$ contains a point $\lambda$ for which the subspace of $\mathbb{C}^{n}$ spanned by the vectors $x$ with $\langle A x, x\rangle=$ $\lambda\|x\|^{2}$ has dimension 2, while a necessary condition is $\sum_{j=0}^{n-2} a_{n-j} e^{(n-j) i \theta} \sin ((j+1) \pi / n)=$ $\sin (\pi / n)$ for some real $\theta$. Examples are given showing that in general these conditions are not simultaneously necessary and sufficient. We then prove that they are if $A$ is (unitarily) reducible. We also establish a lower bound for the numerical radius $w(A)$ of $A: w(A) \geqslant \cos (\pi /(n+1))$, and show that the equality holds if and only if $A$ is equal to the $n$-by- $n$ Jordan block.

Mathematics subject classification (2010): 15A60.

Keywords and phrases: Companion matrix, numerical range, Crawford number.

\section{REFERENCES}

[1] C.-C. Chang, H.-L. Gau, Y.-S. Wang, S.-C. Wu And Y.-T. YeH, Matrices with defect index one, Oper. Matrices 7 (2013), 865-885.

[2] S. H. ChENG AND N. J. Higham, The nearest definite pair for the Hermitian generalized eigenvalue problem, Linear Algebra Appl. 302/303 (1999), 63-76.

[3] C. R. Crawford, A stable generalized eigenvalue problem, SIAM J. Numer. Anal. 13 (1976), 854 860; Errata, SIAM J. Numer. Anal. 15 (1978), 1070.

[4] M. R. Embry, The numerical range of an operator, Pacific J. Math. 32 (1970), 647-650.

[5] H.-L. GAU, Numerical ranges of reducible companion matrices, Linear Algebra Appl. 432 (2010), $1310-1321$.

[6] H.-L. GaU AND P. Y. WU, Numerical range of $S(\phi)$, Linear Multilinear Algebra 45 (1998), 49-73.

[7] H.-L. GAU AND P. Y. WU, Companion matrices: reducibility, numerical ranges and similarity to contractions, Linear Algebra Appl. 383 (2004), 127-142.

[8] H.-L. GAU AND P. Y. WU, Numerical ranges of companion matrices, Linear Algebra Appl. 421 (2007), 202-218.

[9] U. HAAGERUP AND P. DE LA HARPE, The numerical radius of a nilpotent operator on a Hilbert space, Proc. Amer. Math. Soc. 115 (1992), 371-379.

[10] R. A. Horn And C. R. Johnson, Matrix Analysis, 2nd ed., Cambridge University Press, Cambridge, 2013.

[11] R. A. Horn And C. R. Johnson, Topics in Matrix Analysis, Cambridge University Press, Cambridge, 1991. 
[12] M. MARCuS AND B. N. ShURE, The numerical range of certain 0,1-matrices, Linear Multilinear Algebra 7 (1979), 111-120.

[13] G. Polya And G. Szegő, Problems and Theorems in Analysis, Vol. II, Springer, Berlin, 1976.

[14] G. W. STEWART, Perturbation bounds for the definite generalized eigenvalue problem, Linear Algebra Appl. 23 (1979), 69-85.

[15] K.-Z. WANG, P. Y. WU AND H.-L. GAU, Crawford numbers of powers of a matrix, Linear Algebra Appl. 433 (2010), 2243-2254. 